\title{
A microbiologist's perspective
}

There is much pride in seeing the Journal 25 years on. The science of medical microbiology has developed and expanded more rapidly and more widely than we dreamed possible in the middle of the present century. In 1955, I was instructed to read the 4th edition of Topley and Wilson and the 9th edition of Mackie and McCartney. Successive editions of these texts have influenced many of us. If such comprehensive texts remain feasible in the 1990s, it is interesting to consider the developments that they will reflect and that our writers will take for granted.

\section{Biomedical sciences}

In the 1950s, microbiologists were getting to grips with biochemical pathways that were unwinding and revealing their secrets to biochemically competent colleagues more quickly than I could grasp and assimilate. Molecular Biology entered the stage and, in common with Microbial Genetics, drove our understanding of nucleic acids and genetic exchange mechanisms, of plasmids and insertion sequences and transposons, several wavelengths on. We were expected to know all about triplet codes and frameshifts by this new brigade of hybrid scientists who charmed bacteriologists with their talk of sticky ends and ligation and ever more daunting jargon.

Phage experts brought packets of complicated information to us for consumption. Our colleagues in virology smiled wryly as we struggled to comprehend such things as lysogenic conversion, structural models of fearful icosahedral symmetry, restriction endonucleases and the emerging interferons.

We were similarly assailed by the immunologists who, until the mid-century, had offered us simple concepts of precipitation and agglutination, albeit complicated by prozone phenomena and anamnestic reactions, while they preserved the mystique of their black art in the mists of complement and avidity and such things as the Prausnitz-Küstner reaction and the Koch phenomenon. Suddenly, they too achieved escape velocity and bewildered us further with new families and subsets of cells, a torrent of cytokines, and cascades of interacting factors that challenged even the ability of the haematologists who were rapidly cornering that part of the market.

\section{New equipment}

The biochemists, to some extent pushed aside by these developments, had not been idle. They pooled their intellectual and technical resources and helped to fashion the powerful tools and new approaches to cell research and subcellular analysis that were to transform our laboratories. Fractionating columns and ion-exchange systems became commonplace. Proteins and other biologically important molecules could be separated and purified. Chromatography came into its own, in all its new forms, and electrophoretic procedures produced magic lines. Absorption spectra could be determined and plotted automatically in a fraction of the time that I took in the 1960s to do a simple run manually. Centrifuges became more sophisticated, and ultracentrifuges whirled in most laboratory side-rooms. Special radiolabel counting equipment made quantitation easier, and cell-sorting and counting machines provided invaluable results. Even balances became more user-friendly. With the new breed of fermenters, we could grow large volumes of test cultures, and new methods were conceived for splitting cells to reveal their contents. The electronmicroscope was exploited in parallel with this work to relate cell function to structure and to demonstrate the site of some antigen-antibody reactions. Biochemical tests for characterising bacteria multiplied and were incorporated into commercial kits that simplified so much of the work. Sensitivity and specificity became keywords in our detection systems, and quality control began to make its mark. There was a time when everything was going to depend upon detection by complement-fixation. Then it was specific immunofluorescence. Then ELISA was to be the universal solution. Now the order of the day is the polymerase chain reaction. Each of these developments has made and continues to make a remarkable contribution. At a more humble level, automatic pipettes suddenly made my magic with a glass pipette, a rubber tube and a mouthpiece seem archaic and unacceptably dangerous.

\section{New rules}

At a blow, Howie took us to task for accepting so much carelessness for so long and obliged us to put our microbiological houses into order. There was a groundswell of safety consciousness that wrecked many time-honoured practices and drove us belatedly to come to terms with the demands of the twentieth century. This had a knock-on effect, thankfully, into hospital infection control-for so long denied adequate resources or status. It is a hard fight, and the combination of office work, specimen collection and 
tea-room facilities that typifies the scene in some persisting hospital ward side-rooms still calls for urgent attention.

The above developments proceeded pari passu with the evolution of better autoc' ves, better disinfectant policies, better control of inflammable and toxic chemicals, better rules on protective clothing and eye shields, and the advancing design of safety cabinets for microbiological work. We had to produce safety manuals and to audit procedures which, in the past, had often slipped haphazardly into potentially dangerous practices.

\section{Antiseptics and disinfectants}

Meanwhile, antimicrobial chemicals were on the move. The flavines gave way to iodophor preparations and chlorhexidine. Boracic acid yielded to hexachlorophane which was abused and lost the commercial fight. Formaldehyde gave way partly to glutaraldehyde. Even ethanol lost to isopropanol. We came to terms with the hypochlorites and the quaternary ammonium compounds, and we had difficult dalliances with agents as diverse as ethylene oxide, $\beta$ propiolactone and the peracids.

\section{Antimicrobial drugs}

The antibiotics would not let us settle. As soon as we had an established set of antimicrobial drugs with which we felt reasonably comfortable, the bacteria changed their sensitivities and the inexorable march of bacterial drug resistance made it necessary to think again. The pharmaceutical companies performed remarkably, often getting brickbats for their commercialism without many plaudits for their enterprise and the major investments in research and development that lay behind their successes. The clinical microbiologists had to cope with variations on the sulphonamide theme, the proliferation of the penicillins, and the sequential replication of the aminoglycosides. Chloramophenicol did not believe in having a family, but a few tetracycline offspring flourished. The cephalosporins virtually ran out of control across four generations, and the macrolides shook off the reins and began to multiply. We were also expected to know about all of the other shots in the locker, from nitrofurantoin to fusidic acid, from rifampicin to the glycopeptides, and from the lincomycins to metronidazole and the other nitroimidazoles. When we thought that there might be a lull, the quinolones were upon us. The mycobacteria, led by the tubercle bacillus but bettered by its atypical fellows, forced us to bear resistance in mind and to extend our knowledge into the realms of para-aminosalicylic acid, isoniazid, pyrazinamide, rifampicin, ethambutol, ethionamide and cycloserine. This was difficult homework.

The antifungal agents clamoured for attention in a hitherto neglected field, with nystatin and amphotericin B, and 5-fluorocytosine, and the imidazoles and griseofulvin requiring us to be aware of their indications and contra-indications.

The antiviral agents, until recently not exciting in their limited clinical usefulness, jumped into prominence with acyclovir's activity against herpes simplex and herpes zoster, and zidovudine's role in the management of AIDS. Amantadine and rimantadine continued to be of prophylactic and therapeutic interest against influenza $\mathrm{A}$, but many of us were uncertain about the use of these anti-flu agents.

Malaria kept us uncertain with its ability to counter our prophylactic and therapeutic strategies. Giardiasis and amoebiasis yielded to metronidazole's challenge. The development of praziquantel brought new hope in the fight against schistosomiasis, and ivermectin showed promise for the control of onchocerciasis. Our awareness of the importance of Pneumocystis carinii as an opportunist pathogen brought pentamidine into our sights and gave new life to co-trimoxazole.

\section{Immunisation}

We were of course expected to keep pace with preventive strategies. Practical immunisation schedules for the protection of our children against infections saw significant and very progressive changes over these years. The unexpected global success of the relatively crude smallpox vaccine and our subsequent ability to discontinue routine smallpox vaccination was a huge encouragement. The victory against poliomyelitis, at least in the developed countries, is wonderful, even if constant vigilance is still essential and the problems of vaccine delivery in many developing countries continue to tax organisational ingenuity. The control of rubella proved difficult. The advent of the combined mumps, measles, rubella (MMR) vaccine holds much promise, with perhaps a few difficulties to be resolved. The pertussis debate preoccupied and worried us for two decades while confusion was stirred on both sides of the argument by some observers and commentators who could have been more careful. The toxoid vaccines against diphtheria and tetanus endorsed our faith in them, while BCG had and still has a very significant but chequered career in global terms. Immunisation has not done well against cholera, but it has been effective against typhoid; new developments in this field will have to do well to justify their general introduction. The production of the crippled oral Ty21a vaccine is an intriguing step along the way. Effective vaccines against malaria and against AIDS are earnestly hoped for, while problems in their development still abound. A vaccine against bacillary dysentery has not gained acceptance, and an effective herpes simplex vaccine eludes us (despite claims to the contrary).

In our awareness of the role of passive immunisation, we were encouraged to know that the immunologists had transformed the rhesus scene and that passive protection can be of very significant help in the prevention or moderation of some viral infec- 
tions and some bacterial challenges. In this context, we still look hopefully at the possible immunological moderation of septic shock.

\section{New developments and new disease presentations}

The learning curve from our general awareness of so-called serum hepatitis, through our recognition of the Australia antigen, and on to our appreciation of the terrible nature of hepatitis B and the first of the hepadnaviruses, was remarkably steep in the 1960s and 70s. These were alarming years, perhaps conditioning us for what was still to come. At one point, it looked as if other exotic viruses were going to be even more dramatic threats. Before smallpox was controlled, we had two very worrying misadventures with the virus in Britain that underlined the need for our emerging safety rules. Meanwhile, the viral haemorrhagic fevers were recognised and Lassa, Ebola and Marburg hit the headlines. We were obliged to consider laboratory containment with a vengeance and to set down very strict guidelines for our laboratory handling of dangerous pathogens.

In the 1980s, the human immunodeficiency virus (specifically HIV-1) distracted and unbalanced our laboratories in their diagnostic work and in their research and development programmes. Enough has been said about AIDS elsewhere to make the point. We are still moving into the wood, but spectacular advances have been made in this critically important area of our discipline.

What other new concepts, new diseases, or new presentations of old enemies have confronted us since the 1950s?

The hospital staphylococcus was frightening us with its virulence and its transmissibility in the wards and operating theatres when I began to specialise. Then the coagulase-negative staphylococci demonstrated their opportunism as surgeons advanced their implantation technology and their use of prostheses. When we thought that we were winning against the hospital staphylococcus, strains with multiple antibiotic resistance emerged and we began to run out of antibiotic options to control them. Paradoxically, a new coagulase-negative staphylococcus that caused urinary tract infections in women was given the epithet saprophyticus.

Coliform organisms were competing for first place in the opportunist league as the hospital staphylococcus problem seemed to be abating. We had to come to terms with an apparently infinite range of genera and species from Enterobacter to Branhamella (now Moraxella) and from Klebsiella aerogenes to Acinetobacter calcoaceticus. The variations in the clinical presentation of such challenges seemed to be equally endless. Names of species and genera were changed, meanwhile, by well-meaning taxonomists and this added further to the difficulty of keeping up. Pseudomonas pyocyanea became aeruginosa, for example; this organism, hitherto regarded as a nuisance in genito-urinary work and orthopaedic or burns units, shot into prominence with the expanding respiratory bacteriology of cystic fibrosis.

The pace on the service side quickened further. Streptococcus pyogenes, fully sensitive to penicillin, was far from being beaten and reminded us periodically of its potential for harm with some fulminating infections. Studies on the organism that we had known for years as Streptococcus viridans revealed that there is a viridans complex of species with different ecological niches, and we had to get to know them. In parallel with this, our blood culture technology had to be very significantly improved. The Group B streptococcus, for long regarded rather benignly by medics if not by vets as Streptococcus agalactiae was found to be highly pathogenic for young babies and their puerperal mothers. The Group D streptococci, not to be outdone, showed their paces and their innate resistance to our routine drugs. To add to the complexities in this area, the anaerobic cocci challenged us and our patients in ways that we had not fully appreciated.

The neisseriae ran amok, with the gonococcus attacking across the world and literally in unexpected places; worryingly resistant variants made treatment less straightforward. The other sexually transmitted infectious agents, from herpes simplex viruses to the chlamydiae, and from Haemophilus ducreyi to papilloma viruses and Candida albicans and Trichomonas vaginalis and HIV-1, extended our ability to cope in terms of diagnosis, treatment and understanding. Gardnerella vaginalis and vaginosis presented problems. Bacteroides and anaerobic cocci and chlamydiae and pelvic inflammatory disease made life difficult.

The other major neisserial pathogen, the meningococcus, went on the rampage and frightened us all. There were new or newly recognised frights in the form of the legionellae, the salmonellae, and the listeria story. We had to learn to take care before answering telephoned enquiries from the media, and we had to develop a sixth sense about adverse publicity and litigation as the possible rewards for sincere attempts to inform.

The concept of atypical pneumonia was born with the recognition of Mycoplasma pneumoniae as a respiratory pathogen and the radiological appearances that it produced. With our extending awareness of the ornithoses, the term then embraced chlamydial pneumonitis. Then the pneumonitis of $\mathrm{Q}$ fever was added to the list. The term has gone on, subject to the permissiveness of writers and editors, to be applied to pneumonias caused by some other organisms such as legionellae, klebsiellae, staphylococci and even the tubercle bacillus in some of its presentations. Thus, a term that had a relatively specific connotation when it was coined, has become progressively devalued. The same happened with the atypical or anonymous mycobacteria and we have been driven to find better collective words in our microbiological lexicon as various distinctions become more critical. Similarly, 
and happily, the "non-A-non-B hepatitis" jargon is being resolved as our knowledge increases.

The acknowledged range, versatility and pathogenic potential of the anaerobes widened as we learned to be more effective in culturing and characterising members of the clostridia, bacteroides, fusobacteria and anaerobic cocci. It is not enough now to refer to the group previously known as bacteroides, because this has split into several genera. Patients with conditions ranging from cerebral abscess to peritonitis, or from lung abscess to pelvic inflammatory disease, are the better for these advances in our technical ability and our applied knowledge.

The salmonellae proliferated in our media (in all senses). The shigellae were relatively quiet on the home front until very recently, but Escherichia coli showed how it could run the whole gamut from a classically harmless commensal to a vicious cholera-like pathogen, or a shigella-like agent, or a virulent salmonella. My colleagues in our diagnostic laboratories were besieged by clamant clinical requests for help on all of these sectors, while our academic superiors chided them for not doing more research. The campylobacters rubbed salt in the wounds of our pride by demonstrating the duration and extent of our ignorance in the years before their discovery, and the helicobacters are presently turning the knife in the direction of our surgical and medical colleagues.

The range and diversity of food-borne infections and intoxications are not yet fully known and the subject is already vast. Our understanding of infectious diarrhoea had to make rapid progress and still needs to be actively advanced. The yersiniae asked to be recognised as enteropathogens, and Cryptosporidium is currently difficult to ignore (despite the head-in-thesand attitude that many of us adopted in the 1980s). Only a few years ago, we knew virtually nothing about pseudomembranous colitis and Clostridium difficile.

At present, it is difficult to write definitively about traveller's diarrhoea. The cholera vibrios continue to defeat our best efforts, although the clinical management of cholera has advanced spectacularly, and typhoid is still a serious world problem. In the global context, tetanus and botulism and rabies and diphtheria and a host of other infections are still killers. These latter topics must relate more to our teaching and travels than to our experience in Britain over the years, but travel and travellers move ever more quickly in our time and microbiologists are expected to know what is happening across the world. Lyme disease merits distinguished mention here as a recent addition to the extending list of newly recognised infections that have been so well and so rapidly investigated and written up.

\section{An unusual new challenge}

The mad cows caught us unawares and in no mood to entertain the prospect of rewriting major textbook sections on everything from basic rules of sterilisation to fundamental concepts of infection and infectious disease. Bovine spongiform encephalopathy broke upon the scene like a bombshell, and the new notion of prion disease had us scuttling to the libraries and asking our veterinary colleagues and our neuropathologists if the microbiological earth was flat after all. We are still coming to terms with this one. It is difficult to work from first principles when the evidence suggests that first principles cannot apply.

\section{New teamwork}

These developments were made possible by new teamwork across many disciplines and at many levels. Medical, dental, veterinary and science graduates joined forces with skilled laboratory workers to make the advances happen. Infection Control Nurses joined the team, with statisticians and epidemiologists to work on the data that we produced and to indicate when significant trends called for positive action. The setting up of Communicable Disease Centres was a major advance. The concept of the Medical Laboratory Scientific Officer flourished and a proper career structure was forged, though it has to be recorded that a proper career structure for many of our most effective post-doctoral colleagues is still far from being assured. These have been hard productive years.

\section{New lines of communication}

The computer and related technology transformed our day-to-day work over the quarter-century. Data storage and retrieval, the sequential development of a laboratory report, work-sheet practices, surveillance and audit, personnel management, stock control and financial control were all made more efficient (or should have been). The challenge was to abandon the old laborious systems and bring in the new hardware and software while training the teams and finding the money and keeping the show on the road. Many laboratories deserve much credit for this remarkable sea-change in a relatively short time. It may be some comfort to its contributors to know that the Journal and its editors and staff and publishers were similarly overwhelmed with the same wave that left no recording system unchanged.

\section{Postscript}

As I write, the pneumococcus and Haemophilus influenzae have survived the antibiotic era with remarkable ease. It is winter, and they remind me that antibiotic sensitivity isn't everything, even though these organisms can turn that trick too. Our major difficulty continues to be that, despite so much effort, our fundamental knowledge of mechanisms of microbial pathogenicity is still inadequate. We have made major advances in elucidating the pathogenicity of some pathogens, such as the anthrax bacillus, and in explaining the organotropism of Brucella abortus, for 
example. We know a lot about the mode of action of diphtheria toxin, a fair amount about the various bacterial cytolytic toxins and cholera toxin, and some important facts about the toxins that produce tetanus and botulism. We have important leads on adhesion mechanisms that seem to allow various pathogens access to their chosen host cell surfaces-and we are beginning to gain insight into the switches that might then operate to allow an invader to upset the internal control systems of the host cell. The virologists are well ahead of the bacteriologists in this sector and they must be frustrated that it is difficult to exploit the information therapeutically or prophylactically in all but a few models so far. The as yet unfulfilled promise of the interferons is galling, although an admittedly limited application is encouraging. The success of acyclovir is inspiring.

There is much to do and to record in the next 25 years. The Journal's role in carrying our microbiological flag is internationally acknowledged and highly regarded. It has been a privilege to be one of its editors, and a joy to see it prosper.

J. G. COLleE 204 Newhaven Road, Edinburgh EH6 4QE. 